\title{
Modal Aspects of Object Types and Part-Whole Relations and the de re/de dicto Distinction
}

\author{
Giancarlo Guizzardi \\ Federal University of Espírito Santo (UFES), Vitória, Brazil \\ Laboratory for Applied Ontology, ISTC-CNR, Trento, Italy \\ guizzardi@loa-cnr.it
}

\begin{abstract}
In a series of publications, we have proposed a foundational system of ontological categories which has been used to evaluate and improve the quality of conceptual modeling languages and models. In this article, we continue this work by employing theories from Formal Ontology, Cognitive Psychology and Philosophical Logic to systematically investigate some important modal aspects of the ontological categories represented in structural conceptual models. In particular, we focus on Object Types and Part-Whole Relations, formally characterizing some modal properties that motivate the proposal of a number of distinctions within these categories. In addition, we show how two types of modality known in philosophical logic (de re/de dicto modality) can be used to address some subtle issues that appear in conceptual diagrams when different sorts of object types and part-whole relations are combined.
\end{abstract}

\section{Introduction}

In recent years, there has been a growing interest in the application of Foundational Ontologies, i.e., formal ontological theories in the philosophical sense, for providing real-world semantics for conceptual modeling languages, and theoretically sound foundations and methodological guidelines for evaluating and improving the individual models produced using these languages. This increasing interest can be noticed by the growth of the number of publications dedicated to the subject, including books [13], journal issues [17] and articles published at this forum [1,6]. However, by looking at these publications, one may notice that there is an issue of substantial importance in Formal Ontology but which has been given relative little attention in that community, namely, the examination of the modal properties of the ontological categories represented in the constructs of these languages.

In this article we continue our work on developing ontological foundations for conceptual modeling [4-6]. The objective here is to employ theories from Formal Ontology, Cognitive Psychology and Philosophical Logic to systematically investigate some important modal properties of structural conceptual models. In section 2, we give a brief presentation of a system of Quantified Modal Logics used in the remaining sections. In section 3, we revisit our theory of Object Types (e.g., Kinds, Roles, States, Mixins) presented in [6] focusing on some modal aspects of these categories, and formally characterizing these aspects with the system presented in section 2 . 
In section 4, we revisit a theory presented in [4] elaborating on distinctions between mereological (parthood) relations motivated by different modal properties governing the relations between parts and wholes ${ }^{1}$. In section 5 , we present the main contribution of this paper, namely, to formally elaborate on some subtle issues regarding the distinction between de re and de dicto modality, which are manifest in conceptual diagrams when the categories presented in section 4 and 5 are combined. Section 6 briefly discusses related work. Finally, section 7 presents some final considerations.

\section{A Brief Presentation of a System of Quantified Modal Logics}

In order to present a formal characterizations of the notions discussed in this article we make use of a language $L$ of quantified modal logics with identity. The alphabet of L contains the traditional operators $\wedge$ (conjunction), $\vee$ (disjunction), $\neg$ (negation), $\rightarrow$ (conditional), $\leftrightarrow$ (biconditional), $\forall$ (universal quantification), $\exists$ (existential quantification), with the addition of the equality operator $=$, the uniqueness existential quantification operator $\exists$ !, and the modal operators $\square$ (necessity) and $\diamond$ (possibility). The following holds for these three latter operators: (1) $\diamond \mathrm{A}==_{\text {def }} \neg \square \neg \mathrm{A}$; (2) $\square \mathrm{A}==_{\text {def }} \neg \checkmark \neg \mathrm{A}$ and (3) $\exists$ !x $A==_{\text {def }} \exists y \forall x(A \leftrightarrow(x=y))$.

A Model-Theoretic semantics for this language can be given by defining an interpretation function $\delta$ that assigns values to the non-logical constants of the language and a model structure $\mathrm{M}$. In this language $\mathrm{M}$ has a structure $<\mathrm{W}, \mathrm{D}>$ where $\mathrm{W}$ is a non-empty set of worlds and D is a non-empty domain of objects. The domain D of quantification is that of possibilia, which includes all possible entities independent of their actual existence. Therefore we shall quantify over a constant domain in all possible worlds. Informally, we can state that the truth of formulas involving the modal operators can be defined such that the semantic value of formula $\square \mathrm{A}$ is true in world $\mathrm{w}$ iff $\mathrm{A}$ is true in every world $\mathrm{w}$ ' accessible from $\mathrm{w}$. Likewise, the semantic value of formula $\diamond \mathrm{A}$ is true in world $\mathrm{w}$ iff $\mathrm{A}$ is true in at least one world $\mathrm{w}$ ' accessible from $\mathrm{w}$.

There are alternative interpretations regarding the ontological status of possible worlds and a full discussion of the topic is outside the scope of this article. Here, unless explicitly mentioned, we take worlds to represent maximal states of affairs (states of the world) which can be factual (i.e., obtaining in reality) or counterfactual. An alternative interpretation which also appears in the article is that of worlds as histories, i.e., as causally connected sequences of world snapshots (state of affairs), which again, can be either factual or counterfactual. Moreover, we take all worlds to be equally accessible and therefore we omit the accessibility relation from the model structure. As a result we have the simplest language of quantified modal logic (QS5). For a full presentation of such a system one should refer to [2].

Finally, in order to simplify the presentation of the formulas throughout the article we make use a restricted quantification scheme following the notation proposed in [15]: (i) $(\forall \mathbf{S}, \mathbf{x})$ A and (ii) $(\exists \mathbf{S}, \mathbf{x}) \mathbf{A}$, which can be read as for every instance of $S, A$ holds and there is an instance of $S$ such that A holds, respectively. In other words, (i)

\footnotetext{
${ }^{1}$ The theory proposed in [4] and elaborated in [5] discusses a number of other properties of part-whole relations. Here, due to the scope and objectives of this article we focus solely on modally related properties.
} 
and (ii) are meta-linguistic abbreviations to the formulas $(\forall \mathrm{x} \mathrm{S}(\mathrm{x}) \rightarrow \mathrm{A})$ and $(\exists \mathrm{x} \mathrm{S}(\mathrm{x})$ $\wedge$ A), respectively, i.e., they conform to the so-called Fregean analysis of restricted quantification.

\section{Modal Distinctions in a Theory of Object Types}

In the practice of conceptual modeling, a set of primitives is often used to represent distinctions in different sorts of Object Types (Kind, Role, State, Mixin, among others). However, most conceptual modeling languages do not offer methodological support for helping their uses to decide how to represent elements that denote general terms in a given domain (viz. Person, Student, Red Thing, Physical Thing, Deceased Person, Customer) and, hence, modeling choices are often made in an ad hoc manner. Additionally, an inspection of the literature shows that there has been traditionally much disagreement on the meaning of these categories (for extended discussion on this see [6]).

In [6], we propose a philosophically and psychologically well-founded theory of types for conceptual modeling and a UML modeling profile based on this theory ${ }^{2}$. In the remaining of this section we briefly revisit this theory. However, the focus here is on the modal properties that motivate the distinctions populating this "Typology of Object Types", as well as on the formal characterization of these distinctions using the system of modal logics presented in section 2. In addition, we focus here on a subset of these distinctions, namely, on Kinds, Roles, Phases, and RoleMixins, which are the most relevant ones for the purposes of this article.

The categories forming this typology that we discuss here are depicted in Figure 1.a. As it can be observed, a fundamental distinction between Object Types is made between Sortal and Mixin Types. Sortals are sorts of types that carry principles of identity, individuation and counting for their instances. A principle of identity is a principle for which we can judge whether two individuals are the same. A principle of counting, in contrast, is one that supports individuation and counting of individuals. To illustrate this point, let us make use of the following thought experiment. Suppose someone is presented with a red entity (e.g., a red shirt) at time $t_{1}$ and asked the following question: "Exactly how many red entities do you see in front of you?". Now, suppose that a part (e.g., one sleeve) of this red entity is extracted and destroyed at a time $t_{2}$, and an additional question is asked: "Is the red entity you are seeing now $\left(t_{2}\right)$ the same you saw before (in $t_{1}$ )?" Notice that none of the questions can receive a determinate answer (an answer with a determinate truth-value): (i) Should a red shirt be counted as one or should the shirt, the two sleeves, and two pockets be counted separately so that we have five reds? The problem in this case is not that one would not know how to finish the counting but that one would not know how to start, since arbitrarily many subparts of a red thing are still red; (ii) How can one know if extracting a piece of the entity alters the identity of that entity? How can one know, for example, if having that piece is an essential property of that entity? The problem in both cases is the type Red does not supply principles based on which these questions can be given determinate answers. Now, notice that if (red) entity is replaced in these

\footnotetext{
${ }^{2}$ This theory as presented in [6] can be seen as the conceptual modeling extension of the OntoClean methodology [3].
} 
questions by (red) shirt, determinate answers can be given to all these questions. Types such as Shirt (but also Person, Car, Dog, Student) are examples of Sortal Types. In contrast, types such as Red (but also Thing, Tall, Heavy and Insured Item) are named Characterizing Types, Attributions or Mixins, since they only attribute properties to (characterize) individuals which have already being individuated by sortal-supplied principles.

The statement that the identity of an individual can only be traced in connection with a sortal type, which carries a principle of individuation and identity to the particulars it collects amounts to one of the best-supported theories in the philosophy of language $[10,15]$, and one that finds strong empirical support in cognitive psychology [5]. Moreover, the distinction between sortals and mixins is reflected in natural language in the distinction between common nouns and other general terms (e.g., adjectives, verbs), respectively. Finally, as discussed in $[3,5,6]$, the role of (sortal-supplied) identity principles is explicitly defended in conceptual modeling as a method for deriving stable and ontologically sound taxonomic structures.

A principle of identity must apply to an individual in all possible situations. For this reason, principles of identity must be supplied by types that are also instantiated by their instances in all possible situations, i.e., type whose instances cannot cease to instantiate without ceasing to exist. This meta-property of types is named Modal Constancy or rigidity and can be formally characterized as in the following formula schema:

Definition 1 (Rigidity). A type $\mathrm{T}$ is rigid if for every instance $x$ of T, $x$ is necessarily (in the modal sense) an instance of $\mathrm{T}$. In other words, if $x$ instantiates $\mathrm{T}$ in a given world $w$, then $x$ must instantiate $\mathrm{T}$ in every possible world $w^{\prime}:(\mathbf{1}) . \mathbf{R}(\mathbf{T})=_{\text {def }}$ $(\forall \mathbf{x} \mathbf{T}(\mathbf{x}) \rightarrow \square(\mathbf{T}(\mathbf{x})))$.

We have that only rigid sortals can supply principles of identities for their instances. A rigid sortal type that supplies a principle of identity for its instances is named here a Substance Sortal or a Kind. This notion of Kind as presented here (also sometimes termed Natural Kind) is associated with the notion of Essence in the philosophical literature. More specifically, a Kind is a type defining all the essential properties for the individuals it classifies. Examples of types typically modeled as Kinds include Person, Planet, Gold, Water, Lepidopteron and City.

Within the category of sortals, we also have types that apply to their instances only contingently (i.e., possibly only in certain situations). Examples include types such as Adolescent, Student, Employee, Philosopher, Deceased, Customer and Caterpillar. Sortals that possibly apply to an individual only during a certain phase of its existence are named Phased-Sortals. Contrary to kinds, phased-sortals are anti-rigid types:

Definition 2 (Anti-rigidity). A types $\mathrm{T}$ is anti-rigid if for every instance $x$ of $\mathrm{T}, x$ is possibly (in the modal sense) not an instance of $\mathrm{T}$. In other words, if $x$ instantiates $\mathrm{T}$ in a given world $w$, then there is a possible world $w$ ' in which $x$ does not instantiate T: (2). $\mathbf{A R}(\mathbf{T})=_{\text {def }} \square(\forall \mathbf{x} \mathbf{T}(\mathbf{x}) \rightarrow \diamond(\neg \mathbf{T}(\mathbf{x})))$.

Being anti-rigid, phased-sortals cannot supply a principle of identity for their instances. However, since they are sortals, they must carry a principle of identity, which they inherit from a Kind. Therefore, we have that every phase-sortal PS must be a subtype of Kind such that PS inherits the principle of identity supplied by K. In other 
words, every instance of PS is necessarily a $\mathrm{K}$ and, thus, obeys the principle of identity supplied by K. For example, for an individual John instance of Student, we can easily imagine John moving in and out of the Student type, while being the same individual, i.e. without losing his identity. This is because the principle of identity that applies to instances of Student and, in particular, that can be applied to John, is the one which is supplied by the kind Person of which the phase-sortal Student is a subtype.

If PS is a phased-sortal and K is the Kind specialized by PS, there is a specialization condition $\varphi$ such that $x$ is an instance of PS iff $x$ is an instance of K that satisfies $\varphi$ [15]. A further clarification on the different types of specialization conditions allows us to distinguish between two different types of phased-sortals which are of great importance to the practice of conceptual modeling, namely, Phases and Roles. Phases constitute possible stages in the history of a Kind. Examples include: (a) Alive and Deceased: as possible stages of a Person; (b) Catterpillar and Butterfly of a Lepidopteran; (c) Town and Metropolis of a City; (d) Boy, Male Teenager and Adult Male of a Male Person.

Roles differ from phases with respect to the specialization condition $\varphi$. For a phase $\mathrm{Ph}, \varphi$ represents a condition that depends solely on intrinsic properties of $\mathrm{Ph}$. For instance, one might say that if John is a Living Person then he is a Person who has the property of being alive or, if Spot is a Puppy then it is a Dog who has the property of being less than one year old. For a role Rl, conversely, $\varphi$ depends on extrinsic (relational) properties of Rl. For example, one might say that if John is a Student then John is a Person who is enrolled in some educational institution, if Peter is a Customer then Peter is a Person who buys a Product $x$ from a Supplier $y$, or if Mary is a Patient than she is a Person who is treated in a certain medical unit. In other words, an entity plays a role in a certain context, demarcated by its relation with other entities. This metaproperty of Roles is named Relational Dependence and can be formally characterized as follows:

Definition 3 (Relational Dependence). A type $\mathrm{T}$ is relationally dependent on another type $\mathrm{P}$ via relation $\mathrm{R}$ iff for every instance $x$ of $\mathrm{T}$ there is an instance $y$ of $\mathrm{P}$ such that $x$ and $y$ are related via $\mathbf{R : ~ ( 3 ) . ~} \mathbf{R}(\mathbf{T}, \mathbf{P}, \mathbf{R})=_{\text {def }} \square(\forall \mathbf{x} \mathbf{T}(\mathbf{x}) \rightarrow \exists \mathrm{y} \mathbf{P}(\mathbf{y}) \wedge \mathbf{R}(\mathbf{x}, \mathbf{y}))$.

Mixins (i.e., non-sortals) are types that classify entities that belong to different Kinds, i.e., that obey different principles of identity. As with the category of sortals, mixins can also be rigid or anti-rigid. One type of mixin of great interest in conceptual modeling is the so-called RoleMixin. For example, take the type Insured Item. This type can have as instances entities such as Boats, Cars, Persons, Houses, Work of Art, among others, clearly belonging to different kinds. In addition, instances of this type are only so contingently (an entity can be insured in one situation and not in another one). Finally, an Insured Item is defined in a certain context that includes types such as Insurance Policy and Insurance Agency. Thus, the type Insured Item is an example of a role mixin, i.e., an anti-rigid and relationally dependent mixin.

The discussion of this section is summarized in figures 1.a below. In this figure, we use the notational shortcuts $\mathbf{R}+$ and $\mathbf{R}$ - to represent the meta-properties or rigidity and anti-rigidity, respectively and $\mathbf{D}(-/+)$ to represent the meta-property of relational (in)dependence. In summary, Kinds are rigid, independent sortals that supply a principle of identity for their instances; Phases are independent anti-rigid sortals; Roles 
are anti-rigid and relationally dependent sortals, and RoleMixins are anti-rigid and relationally dependent non-sortals. In this article, we use the stereotypes «Kind», «Role», «Phase», and «RoleMixin» to decorate classes in a UML conceptual model (see figure 1.b) representing these distinctions among object types. It is important to emphasize that UML is used here only for the sake of exemplification, and that the issues addressed here are present in all major conceptual modeling languages.

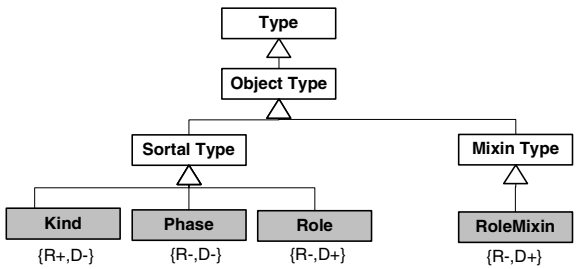

(a)

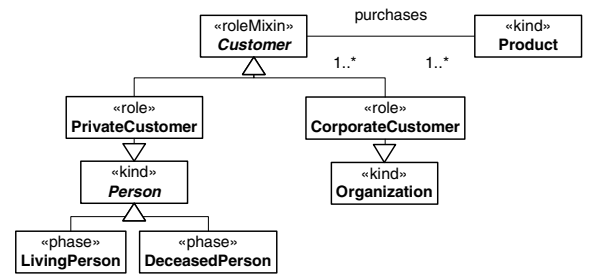

(b)

Fig. 1.a (left) Ontological Distinctions among Fig. 1.b Example of use of a modeling profile Object Types motivated by Modal Meta- based on these distinctions.

Properties;

\section{Modal Distinctions in Part-Whole Relations}

Parthood is a relation of significant importance in conceptual modeling, being present in practically all conceptual modeling languages (e.g., OML, UML, EER). Nonetheless, in many of these languages, the concepts of part and whole are understood only intuitively, or are based on the very minimal axiomatization that these notions require, namely, that of a strict partial order (the so-called Ground Mereology). However, an important aspect to be addressed by any conceptual theory of parthood is to stipulate the different status that parts can have w.r.t. the whole they compose. As discussed by [14], many of the issues regarding this point cannot be clarified without considering modality. One of these issues refers to the notion of separability.

In order to formally define separability, we first define some notions related to the topic of ontological dependence. In particular, the relations of existential and generic dependence discussed in the sequel are strongly based on those defined in [8].

Definition 4 (existential dependence). Let the predicate $\varepsilon$ denote existence. We have that an individual $x$ is existentially dependent on another individual $y$ (symbolized as $e d(x, y))$ iff, as a matter of necessity, $\mathrm{y}$ must exist whenever $\mathrm{x}$ exists, or formally (4). $\operatorname{ed}(\mathbf{x}, \mathbf{y})=_{\text {def }} \square(\varepsilon(\mathbf{x}) \rightarrow \varepsilon(\mathbf{y}))$.

With definition 4 we can propose the concept of an essential part as follows ${ }^{3}$

Definition 5 (essential part). An individual $x$ is an essential part of another individual $y$ iff, $y$ is existentially dependent on $x$ and $x$ is, necessarily, a part of $y$ : $\operatorname{EP}(\mathrm{x}, \mathrm{y})=_{\mathrm{def}}$ $\mathrm{ed}(\mathrm{y}, \mathrm{x}) \wedge \square(\mathrm{x} \leq \mathrm{y})$. This is equivalent to stating that $\operatorname{EP}(\mathrm{x}, \mathrm{y})=_{\operatorname{def}} \square(\varepsilon(\mathrm{y}) \rightarrow \varepsilon(\mathrm{x})) \wedge \square$

\footnotetext{
${ }^{3}$ Following [14] we use the symbols $\leq$ and $<$ to represent parthood and proper parthood, respectively, and we have that $(\mathrm{x} \leq \mathrm{y})=_{\text {def }}(\mathrm{x}<\mathrm{y}) \vee(\mathrm{x}=\mathrm{y})$.
} 
$(\mathrm{x} \leq \mathrm{y})$, which is, in turn, equivalent to $\mathrm{EP}(\mathrm{x}, \mathrm{y})=_{\text {def }} \square(\varepsilon(\mathrm{y}) \rightarrow \varepsilon(\mathrm{x}) \wedge(\mathrm{x} \leq \mathrm{y}))$. We adopt here the mereological continuism defended by [14], which states that the partwhole relation should only be considered to hold among existents, i.e., $\forall \mathrm{x}, \mathrm{y}(\mathrm{x} \leq \mathrm{y})$ $\rightarrow \varepsilon(\mathrm{x}) \wedge \varepsilon(\mathrm{y})$. As a consequence, we can have this definition in its final simplification $(5) . \mathbf{E P}(\mathbf{x}, \mathbf{y})=_{\text {def }} \square(\varepsilon(y) \rightarrow(x \leq y))$.

Figures 2.a and 2.b below depict examples of essential parts. In figure 2.a, every person has a brain as part, and in every world that the person exists, the very same brain exists and is a part of that person. In figure 2.b, we have an analogous example: a car has a chassis as an essential part, thus, the part-whole relation between car and chassis holds in every world that the car exists. To put in a different way, if the chassis is removed, the car ceases to exist as such, i.e., it looses its identity.
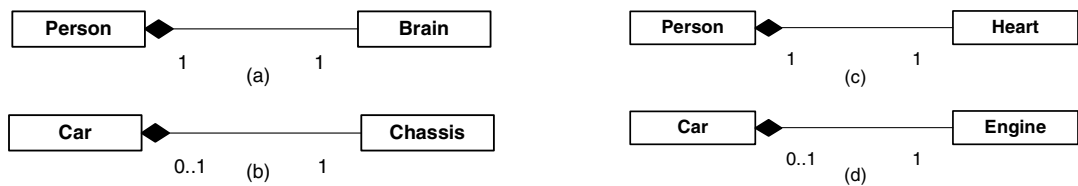

Fig. 2. (a-b) Wholes and their Essential parts; (c-d) Wholes and their Mandatory parts

The UML notation used in figure 2 highlights a problem that exists in practically all conceptual modeling languages. In order to discuss this problem, let us examine the models represented in figures 2.c and 2.d. According to the UML semantics, the models of figure 2.a and 2.c convey exactly the same kind of information. However, this is not the case, in general, in this domain in reality. Typically, the relation between a person and his brain is not of the same nature as the relation between a person and his heart. Differently from the former, a particular heart is not an essential part of a person, i.e., it is not the case that for every person $\mathrm{x}$ there is a heart $\mathrm{y}$, such that in every possible circumstance $\mathrm{y}$ is part of $\mathrm{x}$. For instance, the fact that an individual John had the same heart during his entire lifetime is only accidental. With the advent of heart transplants, one can easily imagine a counterfactual in which John had been transplanted a different heart. An analogous argument can be made in the case of figure 2.d. Although every car needs an engine, it certainly does not have to be the same engine in every possible world.

The difference in the underlying real-world semantics in the cases of figure 2.a and 2.c are made explicit if we consider their corresponding formal characterization. In the case of fig.2.a, since it is a case of essential parthood, we have that: (figure 2.a) $\square((\forall$ Person, $\mathbf{x})(\exists$ !Brain,y $) \square(\varepsilon(\mathbf{x}) \rightarrow(\mathbf{y}<\mathbf{x})))$, whereas in the case of figure 2.c, the corresponding axiomatization is (figure 2.c) $\square((\forall$ Person, $x) \square(\varepsilon(\mathbf{x}) \rightarrow(\exists$ !Heart,y)(y $<\mathbf{x})$ )). A similar distinction can be made for the case of figures 2.b and 2.d: (figure 2.b) $\square((\forall$ Car, $x)(\exists$ !Chassis,y $\square(\varepsilon(\mathbf{x}) \rightarrow(\mathbf{y}<\mathbf{x})))$ and (figure 2.d $) \square((\forall$ Car,x $) \square(\varepsilon(\mathbf{x})$ $\rightarrow(\exists$ !Engine, $\mathbf{y})(\mathbf{y}<\mathbf{x})))$.

In cases such as those depicted in the specifications of figures 2.c and 2.d, an individual is not specifically dependent of another individual, but generically dependent of any individual that instantiates a given type. The concept of generic dependence is defined as follows: 
Definition 6 (generic dependence). An individual $y$ is generic dependent of a type $\mathrm{T}$ iff, whenever $\mathrm{y}$ exists it is necessary that an instance of $\mathrm{T}$ exists. This can be formally characterized by the following formula schema: $(\mathbf{6}) . \mathbf{G D}(\mathbf{y}, \mathbf{T})=_{\operatorname{def}} \square(\varepsilon(\mathbf{y}) \rightarrow$ $\exists \mathbf{T}, \mathbf{x} \varepsilon(\mathbf{x}))$.

We name individuals such as the instances of Heart and Engine in figures 2.c and 2.d, respectively, mandatory parts:

Definition 7 (mandatory part). An individual $x$ is a mandatory part of another individual $y$ iff, $y$ is generically dependent of an type T that $x$ instantiates, and y has, necessarily, as a part an instance of T: (7). MP(T,y) $=_{\text {def }} \square(\varepsilon(\mathbf{y}) \rightarrow(\exists \mathbf{T}, \mathbf{x})(\mathbf{x}<\mathbf{y}))$.

In order to represent the ontological distinction between essential and mandatory parts, we propose an extension to the UML notation used in the examples for the remaining of this paper. We assume that the minimum cardinality of $\mathbf{1}$ in the association end corresponding to the part represents a mandatory part-whole relation. To represent the case of an essential part-whole relation, we propose to extend the current UML aggregation notation by defining the Boolean meta-attribute essential.

When the meta-attribute essential equals true then the minimum cardinality in the association end corresponding to the part must also be 1. This is expected to be the case, since essential parthood can be seen as a limit case of mandatory parthood. When essential equals false, the tagged value textual representation can be omitted. This extended notation is exemplified in figure 3 below.



Fig. 3. Extensions to the UML notation to distinguish between essential and mandatory parts

We emphasize that the particular examples chosen to illustrate the distinction between essential and mandatory parts are used here for illustration purposes only. For example, when modeling brain as an essential part of persons and heart as a mandatory one, we are not advocating that this is a general ontological choice that should be countenanced in all conceptualizations. Conversely, the intention is to make explicit the consequences of this modeling choice, and to advocate for the need of explicitly differentiating between these two modes of parthood. The choice itself, however, is always left to the model designer and is conceptualization-dependent.

Up to this moment, we have interpreted possible worlds as maximal state of affairs, which can be factual or counterfactual. In other words, we have assumed a branching structure of time, and each world is taken at a time interval in a (factual or counterfactual) time branch. An alternative is to interpret possible worlds as histories, i.e., as the sum of all state of affairs in a given time branch. In this alternative conception of worlds, we can examine the possible relations between the lifespan of wholes and parts in different types of parthood relations. For instance, figure 4.a illustrates the possible relations between the lifespan of a whole and one of its essential parts. 

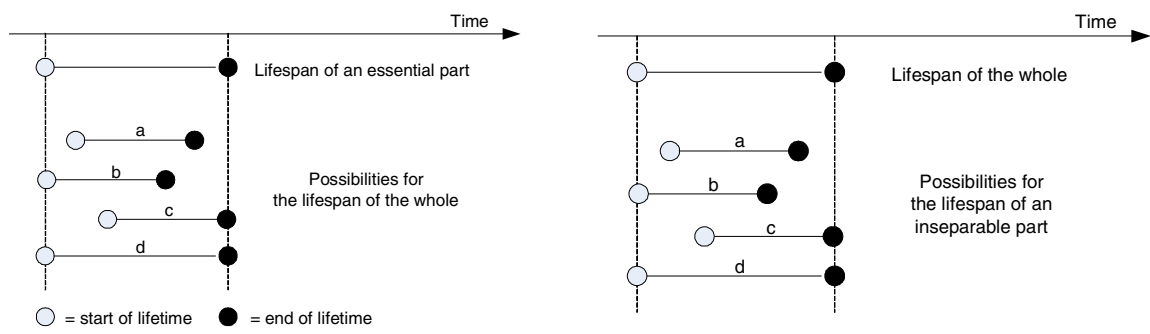

Fig. 4. Possible relations between the life spans of an individual whole and: (a-left) one of its essential parts; (b) one of its inseparable parts.

This figure illustrates the true possibilities for, for instance, the relation between a chassis and a car as depicted in figure 2.b. In this case, the lifetime of the chassis is completely independent from the lifetime of any of the cars it happens to be a part of. Actually, as represented in figure 2.b, a chassis does not even have to be connected to a car (whole). This is a case of, what we term, essential part with optional whole.

Conversely, if we analyze the relation between a brain and a person, we come to the conclusion that the lifespan (d) in figure 4.a is the only real possibility in this case. That is to say that the lifespan of a person and her brain should necessarily coincide. This is because, in this case, a brain is also existentially dependent on its host. Whenever we have the situation that a part is existentially dependent on the whole it composes, we name it an inseparable part:

Definition 8 (inseparable part). An individual $x$ is an inseparable part of another individual $y$ iff, $x$ is existentially dependent on $y$, and $x$ is, necessarily, a part of $y$ : (6). $\mathbf{I P}(\mathbf{x}, \mathbf{y})=_{\operatorname{def}} \square(\varepsilon(\mathbf{x}) \rightarrow(\mathbf{x} \leq \mathbf{y}))$.

The possible relations between the life spans of an inseparable part and its (essential) whole are depicted in figure 4.b. The case of an essential and inseparable part is shown in figure 5 below.

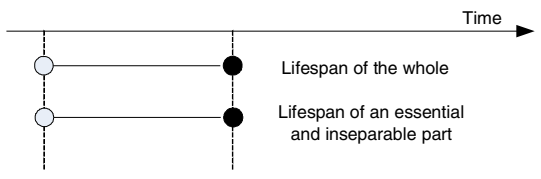

Fig. 5. Possible relations between the life spans of an individual whole and one of its essential and inseparable parts

Figure 4.b does not represent all the possibilities for, for instance, the relation between a heart and its bearer (figure 2.c), since the heart of person is not an inseparable part of a person and, hence, their life spans can be completely independent. A heart can pre-exist its bearer as well as survive its death. Nonetheless, a heart must be part of $a$ person, only not necessarily the same person in all possible circumstances. For these cases, of generic dependence from the part to a whole, we use the term parts with mandatory wholes: 
Definition 9 (mandatory whole). An individual $y$ is a mandatory whole for another individual $x$ iff, $x$ is generically dependent on a type T that $y$ instantiates, and $x$ is, necessarily, part of an individual instantiating T: (7). MW(T,x) $=_{\operatorname{def}} \square(\varepsilon(\mathbf{x}) \rightarrow$ $(\exists \mathbf{T}, \mathbf{y})(\mathbf{x}<\mathbf{y})))$.

Once more, the distinction between inseparable parts and parts with mandatory wholes is neglected in practically all conceptual modeling languages. For this reason, we propose to extend the current UML aggregation notation with the Boolean metaattribute inseparable to represent inseparable parts. When inseparable is equal to true, the minimum cardinality constraint in the association end corresponding to the whole type must be at least $\mathbf{1}$. If inseparable is equal to false, the tagged value textual representation can be omitted. A UML class representing a whole type involved in an aggregation relation with minimum cardinality constraint of at least $\mathbf{1}$ in its association end represents a type whose instances are mandatory wholes.

\section{The de re/de dicto Modal Distinction}

In the previous section, we have presented a distinction between parthood relations w.r.t. ontological dependence containing two possible subtypes: (i) essential parts: characterized by existential dependence from the whole to a part; (ii) mandatory parts: characterized by generic constant dependence from the whole to the type a part instantiates.

As mentioned in the previous section, the relations between a person and her brain, on one hand, and a person and her heart, on the other, can exemplify part-whole relations of sort (i) and (ii), respectively. These two situations taking the human body as an example are depicted in figure 6 together with their corresponding modal logics formalizations. For the sake of simplicity, we formalize in this case only the axioms w.r.t. the relation from the whole to the part. All other axioms are omitted.

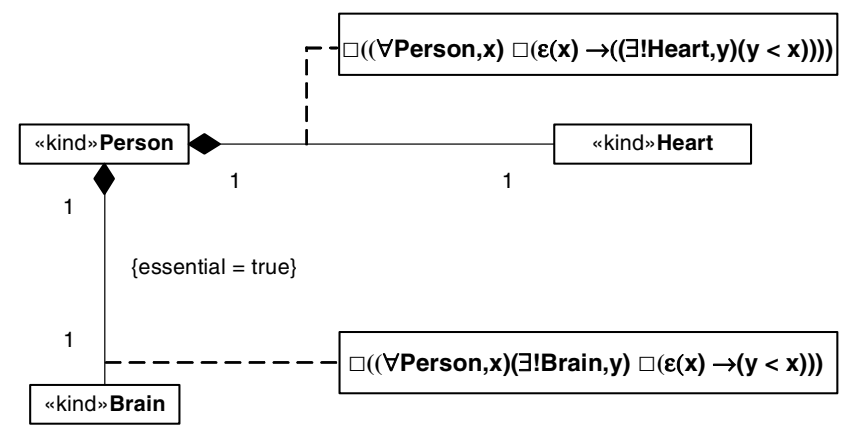

Fig. 6. Representation of essential and mandatory parthood in a model of the human body

In all examples used in section 4, the object types representing wholes are Kinds. Let us now investigate how these different sorts of necessary parthood relations can be used to characterize non-rigid types, such as Roles, Phases or Role Mixins. Suppose, for instance, the situation depicted in figure 7. The figure illustrates the 
relation between a Boxer and one of his hands. What the picture attempts at representing is the statement that "every boxer must have a hand". This relation is certainly not one of mandatory parthood, since it is not the case that a Boxer depends generically on the type hand but specifically on one particular hand ${ }^{4}$. It thus appears to be the case that this relation is one of essential parthood. However, this is not true either. If a hand were to be considered an essential part of a particular boxer then the corresponding formula represented in figure 7 should be valid. To show that this is not the case, suppose the following: let John be a boxer in world w and let $\mathrm{x}$ be John's hand in w. What the formula in figure 7 states is that in every world w' in which John exists, $x$ must be part of John in w'. This formula is clearly falsifiable. One just have to imagine a world w', in which John exists without being a boxer and without having $\mathrm{x}$ as his hand (supposed that $\mathrm{x}$ has been tragically amputated in $\mathrm{w}^{\text {') }}$. This problem arises from the ambiguity of the word "must" in "every boxer must have a hand". Intuitively, the situation that this model intended to express is the valid statement that "For every Person $x$, there is a hand $y$, such that in every world that $x$ is a Boxer, $y$ is a hand of $x$ ".

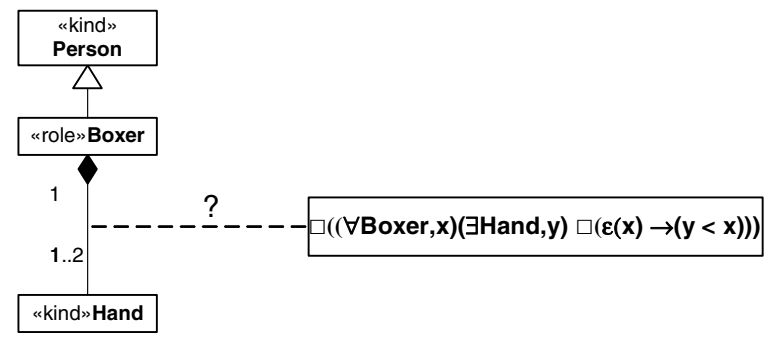

Fig. 7. Problems in the representation of specifically dependent parts for anti-rigid types

In the example of figure 7, Boxer cannot have essential properties and, in particular, cannot have essential parts, since it is an anti-rigid type. In other words, if "to be a boxer" is consider as a property, it is not an essential property itself of any individual. However, this situation can be understood in terms of the philosophical distinction between de re and de dicto modality. Take the following two sentences: (i) The queen of the Netherlands is necessarily queen; (ii) The number of planets in the solar system is necessarily odd. In the de re reading, the first sentence expresses that a certain individual (Beatrix) is necessarily queen. This is clearly false, since we can conceive a different world in which Beatrix decides to abdicate the throne. However, in the de dicto reading the sentence simply expresses that it is necessarily true that in any circumstance whoever is the Dutch queen is a queen. The second sentence works in the converse manner. In the de re reading the sentence (ii) expresses that a certain number (9) is necessarily odd. This is indeed necessarily true. The de dicto reading of the sentence however is false. It is not necessarily the case that the number of planets in the solar system is odd. We can imagine a counterfactual situation in which the solar system has, for instance, 8 or 10 planets. The Latin expressions de re represents a

\footnotetext{
${ }^{4}$ We are here not considering the possibility of hand transplants. Once more, the point of the argumentation is not the specific example.
} 
modality which refers to a property of the thing itself (res), whereas de dicto represents a modality that refers to an expression (dictum). This is made explicit in the logical rendering of the possible readings of these two expressions: (iii-a) de re (false): $\forall \mathrm{x}$ QueenOfTheNetherlands(x) $\rightarrow \square($ Queen(x)); (iii-b) de dicto (true): $\square(\forall \mathrm{x}$ QueenOfTheNetherlands(x) $\rightarrow$ Queen $(\mathrm{x})$ ); (iv-a) de re (true): $\forall \mathrm{x}$ NumberOfPlanets $(\mathrm{x}) \rightarrow \square(\operatorname{Odd}(\mathrm{x}))$; (iv-b) de dicto (false): $\square(\forall \mathrm{x}$ NumberOfPlanets $(\mathrm{x}) \rightarrow \operatorname{Odd}(\mathrm{x}))$.

Take now the expression "every boxer has necessarily a hand". Once more, this expression is true only in one of the readings, namely, the de dicto reading. Whilst it is the case that the expression "In any circumstance, whoever is boxer has at least one hand" is necessarily true, it is false that "If someone is a boxer than he has at least a hand in every possible circumstance". Figure 8, expresses a correct representation of this situation in the de dicto modality.

We now have expressed three different types of dependency relations between wholes and parts: (i) specific dependence with de re modality; (ii) generic dependence with de re modality; (iii) specific dependence with de dicto modality. The remaining option is, of course, conceivable, i.e., generic dependence with de dicto modality. This situation can be captured by the following formula (v) $\square(\forall \mathbf{A}, \mathbf{x} \square(\varepsilon(\mathbf{x}) \wedge$ $\mathbf{A}(\mathbf{x}) \rightarrow \exists \mathbf{y} \mathbf{B}(\mathbf{y}) \wedge(\mathbf{y}<\mathbf{x})))$, in which A represents the (anti-rigid) whole and B represents the part. In this formula, the predicate B is used as what we term here a guard predicate. Intuitively, this predicate "selects" those worlds, in which the parthood relation must hold. The same holds for the predicate Boxer in figure 8 .



Fig. 8. Correct representation of specifically dependent parts of anti-rigid types

We have seen that essential properties, i.e., specific dependence expressed in terms of the de re modality, can only be expressed for rigid types. For anti-rigid types (roles, phases, role mixins), only the corresponding de dicto modality can be applied. Nonetheless, it is also true that for every de re statement regarding an individual $x$, we can express a corresponding de dicto one, by using as guard predicate the substance sortal that $\mathrm{x}$ instantiates. For instance, if it is true that "The number of planets in the solar system (9) is essentially odd" then it is also true that "In any circumstance, if 9 is a number then 9 is odd". We therefore could rephrase the formulas in figure 6 as follows: (vi) $\square((\forall$ Person,x $)(\exists$ !Heart,y $\square(\varepsilon(\mathbf{x}) \wedge \operatorname{person}(\mathbf{x}) \rightarrow(\mathbf{y}<\mathbf{x})))$ and (vii) $\square((\forall$ Person, $\mathbf{x}) \square(\varepsilon(\mathbf{x}) \wedge \operatorname{person}(\mathbf{x}) \rightarrow(\exists$ !Heart,y $)(\mathbf{y}<\mathbf{x})))$. Since Person is a kind (rigid type), everything that is person is necessarily a person. In other words, the predicate person is modally constant, and for every object selected by the universal quantifier, person must be true for this object in every possible world. Consequently, (vi) and (vii) are logically equivalent to their counterparts in figure 6. 




Fig. 9. General representation for Immutable and Mandatory parts

In order to achieve a uniform axiomatization, we therefore propose the following formula schemas depicted in figure 9, which must hold irrespective of the type representing the whole being rigid or anti-rigid sortals. If the type $A$ is rigid then $A(x)$ is necessarily true (if true) and the antecedent $(\varepsilon(x) \wedge A(x))$ can be expressed only by $(\varepsilon(x))$. In this case, the B's are truly essential parts of A's. We refrain from using the term essential part for the cases in which a mere de dicto modality is expressed. Therefore, for the case of specific dependence from instances of anti-rigid types to theirs part we adopt the term immutable part instead. Of course, every essential part is also immutable. Generalization axioms analogous to those in figure 9 can be produced for the case of inseparable and mandatory wholes. Figure 10 depicts a representation of inseparable parts and mandatory wholes, in which guard predicates are included to produce generalizations of the axioms in definitions 6 and 7 that are suitable for the cases of both rigid and anti-rigid types.

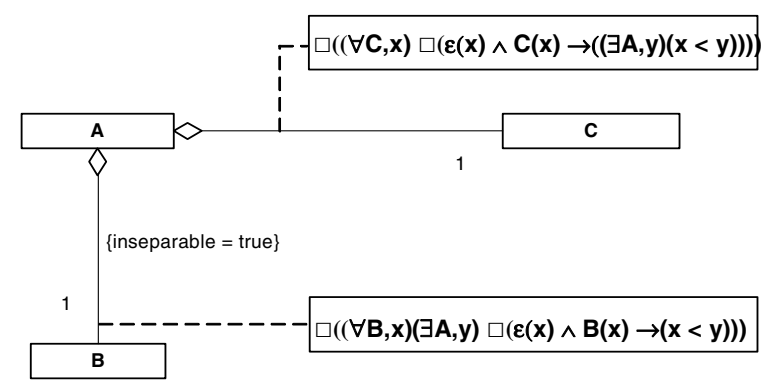

Fig. 10. A general representation scheme for Inseparable Parts and Mandatory Wholes

\section{Related Work}

Two of the works the are closest to ours in objectives w.r.t. establishing a foundation for part-whole relations in conceptual modeling are the pioneering works of James Odell reported in "Six Different Kinds of Composition" [11], and of Brian HendersonSellers and Colleagues reported in a series of articles that includes [7, 12]. There are a 
number of important issues in which our approach differs from these two proposals regarding a number of ontological aspects of part-whole relations (e.g., constitution versus parthood, emergent properties, transitivity of parthood, among others). A fuller comparison between our proposal and these two approaches (among others) can be found in [5]. Here, we concentrate exclusively on the notions directly related to modal aspects of part-whole modeling and, in particular, on the treatment of the notion of separability between parts and wholes.

In his article, Odell has proposed an adaptation of the taxonomy of part-whole relations proposed by Winston, Chaffin and Herrman [16] (henceforth WCH) for the purpose of modeling object-oriented systems. Following $\mathrm{WCH}$, Odell employs a notion of separability as one of the criterion for differentiating between six kinds of partwhole relations. However, this notion employed by Odell (and inherited from WCH) is not a modal notion, but one of physical entanglement. For instance, on page 4 of his article, Odell proposes that the difference between place-area (e.g., EvergladesFlorida) and portion-object (e.g., slice-pie) compositions is that only the former is constituted solely by inseparable parts. Now, if separability is taken in an ontologically meaningful modal sense, there is nothing in the place-area composition relation that requires the parts to be inseparable. For instance, the province of Trentino-Alto Adige is a (place-area) part of Italy, but not an inseparable part, since there are possible worlds (namely before 1921), in which it belonged to the Austrian-Hungarian Empire.

In a different perspective, contra Henderson-Sellers and colleagues [7], we take Lifetime dependency to be a defining feature of those part-whole relations with essential and/or inseparable parts. In this sense, we disagree with examples such as the one used by the authors to justify the existence of parts that are separable, but share the same destruction as the whole: " a car wheel is independent of the car but if the wheel is in the car during the car's destruction then it is also destroyed". In this case, the wheel is clearly separable from the car, it just happened to be the same event that caused the destruction of both objects (had the wheel been separated from the car, the car's destruction would not propagate to the wheel; the wheel can clearly exist in possible worlds in which the car does not exist). In other words, the lifetime coincidence of two separable objects is merely a contingent fact.

This confusion seems to be motivated by an object-oriented programming bias towards conceptual modeling. Traditionally, in OO programming languages, an object can be made responsible for the destruction of other objects as a procedure for memory de-allocation named garbage collecting. Thus, it can be warranted that an object $\mathrm{X}$ should trigger the destruction of other objects coupled with $\mathrm{X}$ in the moment of its destruction, even if the coupling is merely a contingent one.

Finally, it is important to highlight that none of these approaches investigate the modal properties of Object Types. As a consequence, they also do not establish a systematic relation between the different modal properties of part-whole relations and of the object types they are attached to. Here, in contrast, by exposing some subtle ntions that arise when these categories are combined, we can derive practical modeling constraints for the construction of ontologically well-founded conceptual models. 


\section{Final Considerations}

The main objective of this article is to demonstrate the importance of some modal notions in capturing the real-world semantics of some of the conceptual modeling most important constructs, namely, the ones representing the notions of object types and part-whole relations. The article offers a new formal characterization of the modal aspects of the theory originally proposed in [4] by using a systems of quantified modal logics. Moreover, it shows how the formal characterization of the aforementioned ontological categories makes explicit some subtle issues regarding the de re and de dicto modalities in conceptual modeling diagrams which are reflected in two different modes of necessary parthood.

The different types of object types (Kind, Role, Phase and Role Mixin) and partwhole relations (essential, immutable, inseparable and mandatory parts, and mandatory wholes) which result from this analysis, as well as the constraints on how they can be combined (e.g., if a part-whole relation is of type Essential then the whole type must be of type Kind) can be used to analyze and re-design the metamodel of current conceptual modeling languages. An example of an ontologically well-founded language redesigned in this manner is the version of UML proposed in [5]. An example of similar approach towards an extension of ORM using the same ontology proposed in [5] can be found in [9].

It is important to emphasize that the focus of this article is not on aspects of formalization per se but on how some philosophical issues regarding modality can be used to: (i) illuminate the real-world semantics of conceptual modeling constructs; (ii) justify the proposal of more elaborated extensions of these constructs capturing ontological distinctions within the represented categories; (iii) provide some methodological guidelines for helping the user of the language in choosing the most suitable constructs for representing the elements in the universe of discourse according to his own conceptualization. As an example of (iii), if in a given conceptualization the concept Person is taken to be anti-rigid (for instance, in a Legal Ontology, only a conscious entity fully responsible for her acts may be considered to be a person), the model designer knows that this concept should be modeled as a phase, not as a kind. Moreover, since phases are always defined in a phase partition and as a subtype of kind, the designer knows that there are other phases (e.g, UncounciousHumanBeing) that are subsumed by the same kind (e.g., HumanBeing) that are missing in the model. Still on this example, if we have that in two different models a concept represented by the same lexical label (e.g., Person) but with incompatible modal meta-properties (e.g., Person-as-Phase and Person-as-Kind), we have a formal ground for justifying that they are actually different concepts, and for studying what exactly is the relation between them (e.g., Person-as-Kind is equivalent to Human Being). This feature makes an approach such as this one also relevant for the tasks of model integration and semantic interoperability.

\section{References}

1. Evermann, J.: The Association Construct in Conceptual Modelling - An Analysis Using the Bunge Ontological Model. In: Proceedings of 17th CAiSE, Portugal, pp. 33-47 (2005)

2. Fitting, M., Mendelsohn, R.L.: First-Order Modal Logic. Kluwer Publishers, Boston (1999) 
3. Guarino, N., Welty, C.: An Overview of OntoClean. In: Staab, S., Studer, R. (eds.) Handbook on Ontologies, pp. 151-159. Springer, Heidelberg (2004)

4. Guizzardi, G., Herre, H., Wagner, G.: Towards Ontological Foundations for UML Conceptual Models. In: Proceedings of the 1st ODBASE, USA, 2002, p. 1100-1117 ( 2002)

5. Guizzardi, G.: Ontological Foundations for Structural Conceptual Models, PhD thesis, University of Twente, The Netherlands (2005)

6. Guizzardi, G., Wagner, G., Guarino, N., van Sinderen, M.: An Ontologically WellFounded Profile for UML Conceptual Models. In: Proc. of the 16th CAiSE, Latvia, pp.112-126 ( 2004)

7. Henderson-Sellers, B., Barbier, F.: What Is This Thing Called Aggregation? In: proceedings of Technology of Object-Oriented Languages and Systems Europe'99, Nancy, France, pp. 236-250. IEEE Computer Society Press, Washington (ISBN: 0-7695-0275-X) (June 7-10, 1999)

8. Husserl, E.: Logical Investigations, Routledge, London, 1970 (original 1900/1901)

9. Keet, C.M.: Part-Whole Relations in Object-Role Models, International Workshop on Object-Role Modeling (ORM'2006), Montpellier, France, pp.1116-1127 (2006)

10. McNamara, J.: A Border Dispute, the Place of Logic in Psychology. M.I.T. Press, Cambridge (1986)

11. Odell, J.J.: Six Different Kinds of Composition. In: Advanced Object-Oriented Analysis and Design Using UML, Cambridge University Press, New York (1998)

12. Opdahl, A., Henderson-Sellers, B., Barbier, F.: Ontological Analysis of whole-part relationships in OO-models. Information and Software Technology 43, 387-399 (2001)

13. Rosemann, M., Green, P.: Ontologies and Business Systems Analysis, IDEA, USA (2005)

14. Simons, P.M.: Parts. An Essay in Ontology. Clarendon Press, Oxford (1987)

15. van Leeuwen, J.: Individuals and sortal concepts : an essay in logical descriptive metaphysics, PhD Thesis, University of Amsterdam (1991)

16. Winston, M.E., Chaffin, R., Herrman, D.: A taxonomy of part-whole relations. Cognitive Science 11, 417-444 (1987)

17. Wyssusek, B.: On Ontological Foundations of Conceptual Modeling, Scandinavian Journal of Information Systems, Vol.18, No. 1, ISSN 0905-0167 (2006) 